\title{
MODELLING FOR EMERGENCY MANUFACTURING RESOURCES SCHEDULE TO UNEXPECTED EVENTS
}

\author{
Yu, G. D. \\ Department of Industrial and Systems Engineering, National University of Singapore, Singapore \\ E-Mail: iseyg@nus.edu.sg
}

\begin{abstract}
This paper addresses an emergency scheduling for manufacturing resources (ESMR) problem and a method based on event-driven rolling optimization according to the production task priority is developed for solving it. A mathematical model aiming to maximum resource scheduling satisfaction, minimize tardiness penalties and minimize crash cost is proposed firstly. Then, the priority of the tasks in rolling window is analysed based on production system vulnerability. An adaptive multi-objective dynamic resource scheduling algorithm is proposed as a solution of the model above. Analytic results show that it is not only able to effectively reduce the complexity of ESMR, but also to reveal influence of task importance on ESMR. With the analysis, it can provide way of measuring the overall emergency impacts on the systems, and allow the decision-makers to respond to unexpected events that what the best way of resources scheduling is.

(Received in September 2015, accepted in November 2015. This paper was with the authors 1 month for 2 revisions.)
\end{abstract}

Key Words: $\quad$ Emergency Decision-Making, Manufacturing Resources Scheduling, Rolling Optimization, Vulnerability, Resource Scheduling Algorithm

\section{INTRODUCTION}

Unexpected events, such as customer requirements change, material deficiency and equipment failure, are inevitable in production process under unstable and complicated market environment[1]. On this condition, a reasonable and rapid ESMR is a crucial way to reduce emergency impacts on production systems [2]. However, the complex relationship between tasks poses challenges to achieve it. Indeed, a change rarely occurs alone due to the connections within complex systems. A change to a task may trigger chain changes to other tasks, which is called changes propagation [3]. It greatly increases the complexity of the production process. If handled improperly, it could retard the convergence or have a destabilizing effect on the system's behaviour [4]. Thus, the method for ESMR is one of the problems that need to be solved very eagerly.

Though significant research has been conducted, it is still difficult to make a systematically decision under emergency [5]. Some valuable researches are described as below. Johansen and Thorstenson proposed a real-time scheduling policy under urgent orders, respectively [2], but it was on the assumption of the compound Poisson distribution of customers' requirements, which partly weakened the randomness of the emergency. Xia et al. described an emergency management strategy in two-phase linear production system to minimize the deviation penalty cost [6]. Qi et al. studied the resource planning adjustment strategy in supply chain when demand suddenly interrupts $[7,8]$. Jia et al. proposed medical facilities resources scheduling policy under the large-scale emergencies. For frequent incidents in production process [9]. Abreu et al. put forward a mobile handling adjustment technology based on dynamic scheduling scheme to reduce the production time tolerance to disturbance [10]. Zhang discussed the relationship between response levels for emergency and the difficulty of obtaining manufacturing resources in enterprise [11]. Liang et al. proposed a real-time event-driven manufacturing resources conflict resolution method once emergency appeared in operation system [12]. 
By reviewing the achievements above, there is almost local resource scheduling, which optimized the decision result to some extent when emergency appeared. However, a very critical factor, the emergency impacts on the global, is neglected. In fact, tasks iterations, caused by the intricate set of interconnected complex flow is fundamental to production systems. These unexpected events are highly factors that cause the modifications of tasks several times. Thus, on the context of urgency, most of the current researches are local optimum, and the global optimal is not guaranteed when production system is much more complex.

In order to make a reasonable and rapidly ESMR under unexpected events, in this paper, an emergency scheduling model based on event-driven rolling optimization according to the production task priority is developed. Based on the substantial and dramatic new advances in complex networks, the influence of emergencies on the production system is discussed according to production systems vulnerability. Then, the production task priority can be defined combining with the production task priority of time. When emergencies occur, with the goals of maximizing scheduling satisfaction, minimizing tardiness penalties and minimizing the emergency cost, the model for ESMR can be made via the rolling optimization of resources scheduling (RORA), which includes an adaptive multi-objective dynamic resource scheduling algorithm. From the perspective of the global production system, the method above takes the priority to satisfy the most important task for production systems and ensure the production systems coping with emergencies with minimal costs.

This paper is organized as follows: ESMR problem and its model are presented in Section 2. Optimizing strategy including vulnerability-based task importance evaluation and adaptive multi-objective dynamic resource scheduling algorithm are defined in Section 3. Results are illustrated in Section 4. Conclusions are presented in the final Section 5.

\section{PROBLEM DESCRIPTION AND MODELLING}

The ESMR can be described as the following. A production systems contains $n$ tasks, and $T a=\left\{T a_{1}, T a_{2}, \ldots, T a_{n}\right\}$. For unexpected event $\varepsilon$, at least $x_{i m}(\varepsilon)$ number of resource $R_{m}$ should be supplemented to $T a_{i}$. The total number of $R_{m}$ is $D_{m}$, in which there are $d_{i m}(\varepsilon)$ available for $T a_{i}$. The latest arrival time of $R_{m}$ that $T a_{i}$ allows is $L T_{i m}(\varepsilon)$. Besides, each unit of $R_{m}$ will spend $c_{i m}(\varepsilon)$ in this process, and the upper bound of the cost is $C(\varepsilon)$. Based on the aforementioned, the problem is how to calculated the value of the resources supplied $d_{i m}(\varepsilon)$ and delivery time $T_{i m}(\varepsilon)$ of the $R_{m}$, with the highest satisfaction, the lowest emergency cost and the lowest tardiness penalties. Suppose that the satisfaction of $T a_{i}$ to the assigned resources is denoted by frac $_{m i}$ and the importance of $T a_{i}$ to the whole production systems is $\varpi_{i}$, after all parameters uniformed according to Amankwah et al. [13], the model of ESMR can be represented below.

$$
\begin{gathered}
F=\min \left(-\sum_{m=1}^{r} \sum_{i=1}^{n} \varpi_{i} f r a c_{m i}(\varepsilon)\right)+\min \left(\sum_{i=1}^{n} \beta_{i} \cdot \max \left(0, T_{i m}(\varepsilon)-L T_{i m}(\varepsilon)\right)\right)+\min \sum_{i=1}^{n} \sum_{m=1}^{r} c_{i m}(\varepsilon) \\
d_{i m}(\varepsilon) \geq x_{i m}(\varepsilon), \forall i, m \\
\quad \sum_{i=1}^{n} d_{i m}(\varepsilon) \leq D_{m} \\
f r a c_{i m}(\varepsilon)=\alpha \cdot f r a c_{i m, d}(\varepsilon)+\varsigma f r a c_{i m, t}(\varepsilon) \\
\operatorname{frac}_{i m, d}=\left\{\begin{array}{l}
1 \quad d_{i m}(\varepsilon) \geq x_{i m}(\varepsilon) \\
\frac{d_{i m}(\varepsilon)}{x_{i m}(\varepsilon)}, d_{i m}(\varepsilon) \leq x_{i m}(\varepsilon)
\end{array}\right.
\end{gathered}
$$




$$
\begin{gathered}
\operatorname{frac}_{i m, t}=\left\{\begin{array}{l}
1 \quad, L T_{i m}(\varepsilon) \geq T_{i m}(\varepsilon) \\
\frac{T_{i m}(\varepsilon)-L T_{i m}(\varepsilon)}{L T_{i m}(\varepsilon)}, L T_{i m}(\varepsilon) \leq T_{i m}(\varepsilon)
\end{array}\right. \\
\sum_{i=1}^{n} \sum_{m=1}^{r} c_{i m}(\varepsilon) \cdot d_{i m}(\varepsilon) \leq C(\varepsilon) \\
T_{i m}(\varepsilon) \leq T_{j m}(\varepsilon), \varpi_{i} \geq \varpi_{j}, L T_{i m}(\varepsilon)=L T_{j m}(\varepsilon) \\
T_{i m}(\varepsilon) \leq T_{j m}(\varepsilon), L T_{i m}(\varepsilon) \leq L T_{j m}(\varepsilon) \\
T_{i m}(\varepsilon)=S T_{i m}(\varepsilon)+T T_{i m}(\varepsilon)+E T_{i m}(\varepsilon) \\
i \in[1,2, \cdots, n], m \in[1,2, \cdots, r]
\end{gathered}
$$

Here, Eq. (1) is the optimal decision making objective, which means the highest satisfaction, the lowest emergency cost and the lowest tardiness penalties under $\varepsilon$. Eq. (2) denotes the resources assigned should meet the minimum requirements of task starting. Eq. (3) describes all emergency resources should not over upper limit. Eqs. (4) to (6) are constraints about scheduling satisfaction. Eq. (7) expresses the emergency cost constraints. Eq. (8) means that emergency resources should take priority to be assigned to the tasks with a higher degree if they have the same latest start time, namely the priority of importance. Meanwhile, Eq. (9) declares that emergency resources should take priority to be assigned to the tasks with an earlier start time, namely the priority of start time. Eq. (10) shows the constraint of delivery time. $S T_{i m}(\varepsilon), T T_{i m}(\varepsilon)$ and $E T_{i m}(\varepsilon)$ denote the loading time, transport time and unloading time, respectively.

\section{ROLLING OPTIMIZING FOR ESMR}

In this paper, RORA is developed to meet the time requirement. The basic idea is that tasks are divided into serial sub-sets according to their start time, and they are pushed farther forward as time goes on. The tasks sets can be called rolling window. In each resources scheduling, only the tasks in current rolling window will be planned. As the time going, the assigned resources are deleted from the rolling window, and new tasks to assign join in, then the update of rolling window is realized. The RORA decomposed the complex emergency scheduling problem into several static scheduling problems, which reduces the complexity and difficulty of solving the original problem. The process is illustrated in Fig. 1.

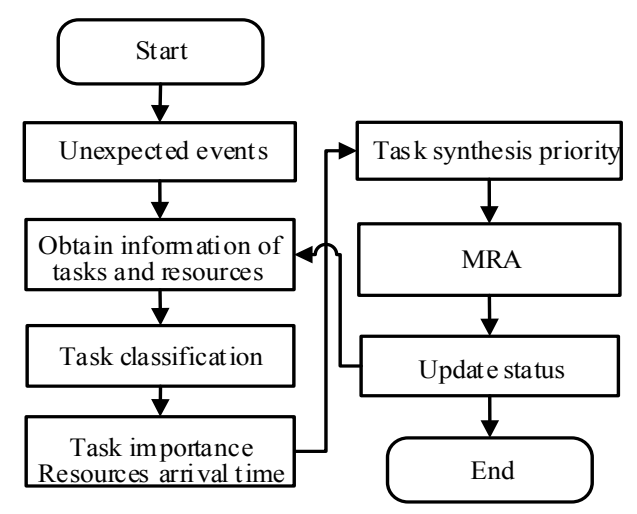

Figure 1: Process of the RORA.

Step 1: Information acquisition. At $t=0$, obtain information of related tasks and resources in the rolling window, such as production task sequence, required resources, lead time of each resource, delivery costs and so on. 
Step 2: Task classification. Based on Step 1, according to the status, the tasks in rolling window can be divided into three types: to be allocated, in scheduling and allocated. Because of the time, the task classification is dynamic, which means that the same task may be defined different category in different classification.

Step 3: Determine the priority of the tasks to be allocated. For the tasks to be assigned recourses have an earlier start time will be allocated first. Emergency resources should take priority to be assigned to the tasks with a higher degree, if they have the same latest start time, as shown in Eqs. (8) and (9).

Step 4: Implement the ESMR based on heuristic algorithm and update the status.

Step 5: Turn into the next rolling window until all tasks and recourses allocated.

\subsection{Vulnerability-based task importance evaluation}

For the RORA, one of the key steps is scheduling the priority of production tasks. It contains two aspects: the one is priority based on start time, and the other one is priority based on importance. The former can be easily cleared by the task information, but the latter is bound to intricacy because of the task interactions. Thus, this section will focus on the task importance evaluation. Indeed, most of the existing evaluation methods are built based on some indexes, which are difficult to be comprehensive and objective [14]. The vulnerability in complex network theory, demonstrated in some other complex systems such as ecosystem, electrical power system and transportation system [4], will be introduced to analyse the task importance. The vulnerability is usually studied via analysing the changes of system performance based on the assumption of task failure. A higher value of the changes means the more important task for the whole system [15].

(1) Complex network model of production systems

Analysis of complex network model of production systems (CNPS) is as follows.

(i) Complex network model of production systems. Suppose that CNPS contains $n$ tasks, $V=\left(v_{1}, v_{2}, \ldots, v_{i}, \ldots, v_{n}\right)$, and $e_{i j}$ represents the connection from $T a_{i}$ to $T a_{j}$, which means the data flow, material flow and other connection flows. A set of values $W=\left\{w_{i 1}, w_{i 2}, \ldots, w_{i n}\right\}$ are real numbers associated with the links, where $w_{i j}$ represents connection strength from $i$ to $j$. Accordingly, CNPS can be constructed as follows.

$$
G=(V, E, W)
$$

Based on [4], adjacency matrix $A=\left\{a_{i j}\right\}$ can be adopted for CNPS topology, where $a_{i j}$ denotes the relations between tasks.

$$
a_{i j}=\left\{\begin{array}{lc}
w_{i j} & \text { if } i \text { and } j \text { are connected with strength } w_{i j} \\
0 & \text { otherwise }
\end{array}\right.
$$

(ii) Adjacency matrix and the weight of CNPS. Actually, there may be many categories of relations between production tasks, such as information flow, energy flow and material flow [16]. Then, $a_{i j}$ should consider all relations. Suppose $\mathfrak{R}=\left\{r_{\kappa} \mid \kappa=1,2, \ldots, \pi_{i}\right\}$ denotes the task relationship set, where $r_{\kappa}$ represent $\kappa^{\text {th }}$ relationship and $\pi_{i}=\|\Re\|$ indicates the number of relationship. Thus, task relationship matrix (TRM) is proposed to represent complicated dependence of CNPS.

$$
T R M=\left\{t m_{i j, \kappa} \mid i, j=1,2, \ldots, n ; \kappa=1,2, \ldots, \pi_{i}\right\}
$$

Here, $t r m_{i j, \kappa}$ denotes the number of relation $r_{\kappa}$ from $T a_{i}$ to $T a_{j}$. Generally, there are many parallel relationships between two tasks. Let $p_{i j, \kappa} \in[0,1]$ indicate the probability of interplay between $T a_{i}$ and $T a_{j}$ based on relation $r_{\kappa}$. Then, 


$$
P=\left\{p_{i j} \mid p_{i j}=1-\prod_{\kappa=1}^{\pi_{i}}\left(1-p_{i j, \kappa}\right)^{t r m_{i, \kappa}}, 1 \leq i, j \leq n\right\}
$$

where, $p_{i j}$ denotes the effect probability of $T a_{i}$ to $T a_{j}$. Obviously, the higher value of $p_{i j}$, the closer between $T a_{i}$ and $T a_{j}$.

In order to determine the value of $p_{i j}$ and $w_{i j}$, based on expert scoring method proposed in [16] to obtain initial data, a triangular fuzzy number (TFN) [17] is introduced to map the clear value of the probability of relationship $p_{i j}$. A finite set of experts is defined as $E x=\left\{E x_{l} \mid l=1,2, \ldots, \Omega\right\}$. Assume that all experts have uniform weight. Meanwhile, the evaluation object set is $\mathfrak{R}=\left\{r_{\kappa} \mid \kappa=1,2, \ldots, \pi_{i}\right\}$, and its evaluation index is $p_{i j, \kappa}$. Linguistic variables set of experts, $\Theta=\left\{\Theta_{\theta} \mid \theta=0,1, \ldots, l-1\right\}$, is pre-defined by an odd number of elements. Suppose that the linguistic assessment information of $r_{\kappa}$ between $T a_{i}$ and $T a_{j}$ from $E x_{l}$ is $\varphi_{i j, k l}$, then $\varphi_{i j, k l} \in \Theta$. And the TFN of $\varphi_{i j, k l}$ can be expressed by Eq. (16).

$$
\varphi_{i j, \kappa \ell}=\left(\varphi_{i j, \kappa \ell}^{L}, \varphi_{i j, \kappa \ell}^{M}, \varphi_{i j, \kappa \ell}^{O}\right)=\left(\max \left(\frac{\theta-1}{l-1}, 0\right), \frac{\theta}{l-1}, \min \left(\frac{\theta+1}{l-1}, 1\right)\right)
$$

where $\varphi_{i j, \kappa \ell}^{L}, \varphi_{i j, \kappa \ell}^{M}, \varphi_{i j, \kappa \ell}^{O}$ mean the upper, mid-value and lower limit of $\varphi_{i j, \kappa l}$, respectively.

Let $\varphi_{i j, k l}$ indicate the linguistic assessment information of $r_{\kappa}$ from all experts, then

$$
\varphi_{i j, \kappa \ell}=(1 / \Omega) \otimes\left(\varphi_{i j, \kappa 1} \oplus \varphi_{i j, \kappa 1} \oplus \ldots \oplus \varphi_{i j, \kappa \Omega}\right)
$$

Define $\varphi_{i j, \kappa}=\left(\varphi_{i j, \kappa}^{L}, \varphi_{i j, \kappa}^{M}, \varphi_{i j, \kappa}^{O}\right)$, thus

$$
\begin{aligned}
& \varphi_{i j, \kappa}^{L}=1 / \Omega \sum_{\ell=1}^{\Omega} \varphi_{i j, \kappa \ell}^{L} \\
& \varphi_{i j, \kappa}^{M}=1 / \Omega \sum_{\ell=1}^{\Omega} \varphi_{i j, \kappa \ell}^{M} \\
& \varphi_{i j, \kappa}^{O}=1 / \Omega \sum_{\ell=1}^{\Omega} \varphi_{i j, \kappa \ell}^{O}
\end{aligned}
$$

Based on the CFCS [18], the fuzzy value can turn into clear according to Eq. (21).

$$
\begin{aligned}
& p_{i j, \kappa}=L+ \frac{\Delta\left[\begin{array}{l}
\left(\varphi_{i, j, \kappa}^{M}-L\right)\left(\Delta+\varphi_{i j, \kappa}^{O}-\varphi_{i j, \kappa}^{M}\right)^{2}\left(O-\varphi_{i j, \kappa}^{L}\right) \\
+\left(\varphi_{i j, \kappa}^{O}-L\right)\left(\Delta+\varphi_{i j, \kappa}^{M}-\varphi_{i j, \kappa}^{L}\right)^{2}
\end{array}\right]}{\left(\Delta+\varphi_{i j, \kappa}^{M}-\varphi_{i j, \kappa}^{L}\right)\left(\Delta+\varphi_{i j, \kappa}^{O}-\varphi_{i j, \kappa}^{M}\right)^{2}\left(O-\varphi_{i j, \kappa}^{L}\right)} \\
&+\left(\varphi_{i j, \kappa}^{O}-L\right)\left(\Delta+\varphi_{i j, \kappa}^{M}-\varphi_{i j, \kappa}^{L}\right)^{2}\left(\Delta+\varphi_{i j, \kappa}^{O}-\varphi_{i j, \kappa}^{M}\right)
\end{aligned}
$$

Here, $L=\min \left\{\varphi_{i, j, \kappa}^{L}\right\}, R=\max \left\{\varphi_{i, j, \kappa}^{R}\right\}, \Delta=R-L$.

Thus, according to Eqs. (14) to (21), $A$ can be calculated.

(2) Task importance evaluation based on vulnerability

Vulnerability was originally applied in ecology, and extended to the Internet, finance [15]. It is generally used to describe the nature of the element failure impacts on the overall system function. For production systems, vulnerability can be understood as the nature of the impacts on the production system efficiency when production task are in failure (paused or interrupted) caused by a sudden factors. And the greater impact, the more important task is. Thus, the vulnerability can be introduced as the index of assessing the task importance. The greatest advantage of the method is that vulnerability is a global variable, which is able to overcome the shortcoming of traditional methods falling into local perspective. Thus, it can 
contribute to obtain much more exact results. According to the definition of vulnerability in [15], it can be defined as Eq. (22):

$$
V\left[G, v_{i}\right]=\frac{\Phi[G]-\Phi\left[G, v_{i}^{*}\right]}{\Phi[G]}
$$

Here, $G$ denotes the normal CNPS. $v_{i}^{*}$ represents the task in failure under an emergency. $\Phi$ is the performance measurement function of $G$. Thus, $\Delta \Phi=\Phi[G]-\Phi\left[G, v_{i}{ }^{*}\right] \geq 0$ means the efficiency loss value before and after the emergency and $\Phi\left[G, v_{i}{ }^{*}\right]$ denotes the vulnerability for $v_{i}, \Phi\left[G, v_{i}^{*}\right] \in[0,1]$.

As shown in Eq. (22), $\Phi$ is the core element of $\Phi\left[G, v_{i}^{*}\right]$. It should not only measure the effect of isolated node failure on network function, also can consider edge failure. Some existing indexes such as connectivity, condensation and toughness still stand some blemishes [19]. Thus, it is necessary to choose the most appropriate $\Phi$ according to the features of CNPS. In this paper, network efficiency is regarded the performance measurement function of CNPS, as shown in Eq. (23).

$$
\xi(G)=\frac{\sum_{i \neq j i, j \in G} p_{i j}}{n(n-1)}=\frac{1}{n(n-1)} \sum_{i \neq j i, j \in G} \frac{1}{l_{i j}}
$$

where $n$ is the number of nodes in $G$ and $l_{i j}$ means the shortest path between $v_{i}$ and $v_{j}$.

Based on the aforementioned, $\Phi[G]=\xi[G]$, so

$$
V\left[G, v_{i}\right]=\frac{\xi(G)-\xi\left(G, v_{i}^{*}\right)}{\xi(G)}
$$

According to the Eq. (24), the importance of each production task can be calculated.

\subsection{Adaptive multi-objective dynamic resource scheduling algorithm}

ESMR involves large scale of data calculation and combination optimization. Thus, it is necessary to seek an appropriate heuristic algorithm. Yu et al. proposed a multi-objective dynamic scheduling algorithm (MODSA) to resolve the dynamic task scheduling problems [20]. Borrowing from the MODSA, an adaptive multi-objective dynamic resource scheduling algorithm (AMODRAA) is developed to realize the dynamic and flexible resources scheduling during the production process. By calling the available resources, AMODRAA can reduce the influence of the incident to the production process. The flow chart of the algorithm is as shown in Fig. 2.

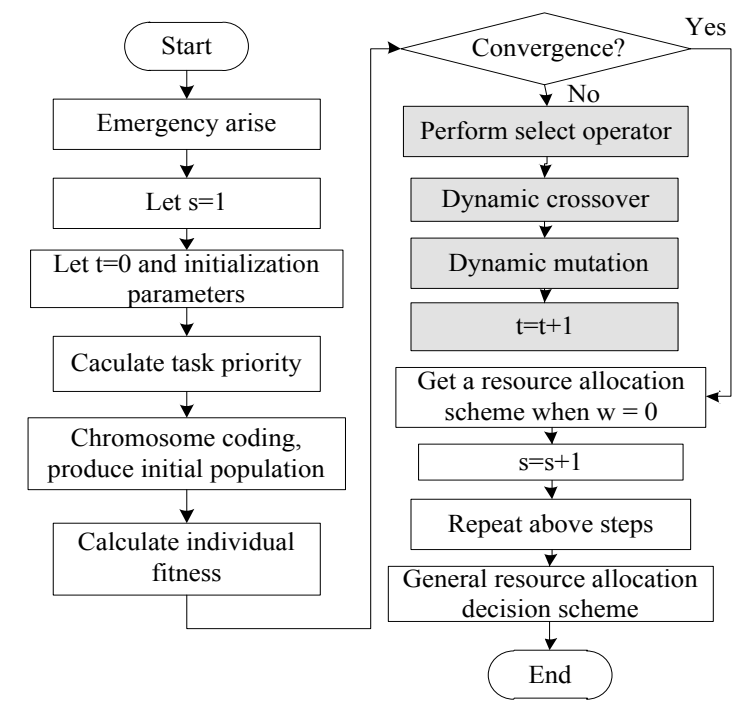

Figure 2: Flow chart of the AMODRAA. 
Step 1: Parameter initialization. Initialize the rolling time $s$ and let $s=1$. At this moment, the first recourse scheduling is conducted. Besides, initialize the time of first task to be assigned, and let $t_{s, a}=0$, and let $t$ denote the evolution algebra of the AMODRAA.

Step 2: Task priority sequence analysis. Combining the information of the tasks to be assigned in rolling window, the priority order can be determined based on Eqs. (8) and (9).

Step 3: Chromosome coding. Suppose that the number of the tasks to be assigned in rolling window is $\rho_{s}$, and a bi-layer coding strategy formed by the fusion of tasks sequences and resources is adopted. The first layer is encoded based on design tasks sequences. The second layer is the resource code corresponding to the first layer of tasks. As shown in Fig. 3, [237514698] means a task sequence in resources scheduling, and the required resources are: $\left[R_{1}, R_{5}, R_{2}, R_{11}, R_{8}, R_{5}, R_{9}, R_{3}, R_{1}\right]$.

\begin{tabular}{|c|c|c|c|c|c|c|c|c|c|c|c|c|c|c|c|c|c|c|c|}
\hline chromosome & 2 & 3 & 7 & 5 & 1 & 4 & 6 & 9 & 8 & \multirow{3}{*}{$\cdots \cdots$} & $3 n$ & $7 n$ & $2 n$ & $5 n$ & 1n & $6 n$ & $8 n$ & $4 n$ & $9 n$ \\
\hline \multirow{2}{*}{$\begin{array}{c}\text { task } \\
\text { resources }\end{array}$} & $\mathrm{Ta}_{2}$ & $\mathrm{Ta}_{3}$ & $\mathrm{Ta}_{7}$ & $\mathrm{Ta}_{5}$ & $\mathrm{Ta}_{1}$ & $\mathrm{Ta}_{4}$ & $\mathrm{Ta}_{6}$ & $\mathrm{Ta}_{9}$ & $\mathrm{Ta}_{8}$ & & $\mathrm{Ta}_{3 \mathrm{n}}$ & $\mathrm{Ta}_{7 \mathrm{n}}$ & $\mathrm{Ta}_{2 \mathrm{n}}$ & $\mathrm{Ta}_{5 \mathrm{n}}$ & $\mathrm{Ta}_{\ln }$ & $\mathrm{Ta}_{6 \mathrm{n}}$ & $\mathrm{Ta}_{8 \mathrm{n}}$ & $\mathrm{Ta}_{4 \mathrm{n}}$ & $\mathrm{Ta}_{9 \mathrm{n}}$ \\
\hline & $\mathrm{R}_{1}$ & $\mathrm{R}_{5}$ & $\mathrm{R}_{2}$ & $\mathrm{R}_{11}$ & $\mathrm{R}_{8}$ & $\mathrm{R}_{5}$ & $\mathrm{R}_{9}$ & $\mathrm{R}_{3}$ & $\mathrm{R}_{1}$ & & $\mathrm{R}_{10}$ & $\mathrm{R}_{51}$ & $\mathrm{R}_{21}$ & $\mathrm{R}_{8}$ & $\mathrm{R}_{3}$ & $\mathrm{R}_{15}$ & $\mathrm{R}_{19}$ & $\mathrm{R}_{31}$ & $\mathrm{R}_{10}$ \\
\hline
\end{tabular}

Figure 3: An example of chromosome.

Step 4: Select operator. This paper uses the roulette wheel method to select the parent chromosomes $i$ with probability $\lambda_{i}$ from the population with popsize individuals.

$$
\lambda_{i}=f_{i} / \sum_{i=1}^{\text {popsize }} f_{i}
$$

Here, $f_{i}$ denotes the individual fitness, and $f_{i}=F_{i}$ according to Eq. (1).

Step 5: Crossover operator. To prevent the operation of algorithm premature and stagnation, this study adopts self-adaptive double point crossover, which is able to increase the population diversity on the basis of making full use of historical information. As shown in Fig. 4.

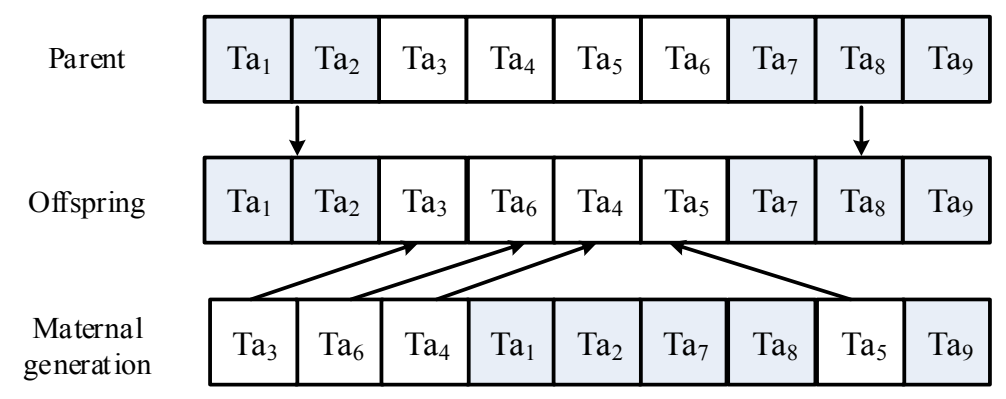

Figure 4: Double point crossover.

Besides, in order to improve the search speed, it is an effective way of timely adjusting the crossover probability in the process of evolution. Thus, in this paper, adaptive adjustment function $\lambda_{c}$ is introduced to improve its ability of dynamic cross in crossover. Let $f_{\max }$ and $f_{\text {avg }}$ denote the maximum and the average individual fitness value respectively. Then,

$$
\lambda_{c}=\left\{\begin{array}{l}
\lambda_{c 1}-\frac{\left(\lambda_{c 1}-\lambda_{c 2}\right)\left(f_{i}-f_{\text {avg }}\right)}{f_{\max }-f_{\text {avg }}}, f_{i} \geq f_{\text {avg }} \\
0, \quad f_{i}<f_{\text {avg }}
\end{array}\right.
$$

Step 6: Mutation. Randomly select a gene according to mutation rate and alter its value, as shown in Fig. 5. Meanwhile, adaptive mutation probability $\lambda_{m}$ is used to improve the convergence speed, and the adaptive adjustment function is in Eq. (27). 


\begin{tabular}{|c|c|c|c|c|c|c|c|c|c|}
\hline Parent & $\mathrm{Ta}_{1}$ & $\mathrm{Ta}_{2}$ & $\mathrm{Ta}_{6}$ & $\mathrm{Ta}_{7}$ & $\mathrm{Ta}_{8}$ & $\mathrm{Ta}_{3}$ & $\mathrm{Ta}_{9}$ & $\mathrm{Ta}_{4}$ & $\mathrm{Ta}_{5}$ \\
\hline Offspring & $\mathrm{Ta}_{1}$ & $\mathrm{Ta}_{2}$ & $\mathrm{Ta}_{9}$ & $\mathrm{Ta}_{7}$ & $\mathrm{Ta}_{8}$ & $\mathrm{Ta}_{3}$ & $\mathrm{Ta}_{6}$ & $\mathrm{Ta}_{4}$ & $\mathrm{Ta}_{5}$ \\
\hline
\end{tabular}

Figure 5: Mutation mechanism.

$$
\lambda_{m}=\left\{\begin{array}{l}
\lambda_{m 1}-\frac{\left(\lambda_{m 1}-\lambda_{m 2}\right)\left(f_{\max }-f^{*}\right)}{f_{\max }-f_{\text {avg }}}, f^{*} \geq f_{\text {avg }} \\
\lambda_{m 1}, \quad f^{*}<f_{\text {avg }}
\end{array}\right.
$$

In Eq. (27), $f^{*}$ denotes the fitness value of the individual in variation.

Then let $t=t+1$, conduct loop iteration until meeting the termination conditions described in the Step 7. Let $s=s+1$, the general ESMR can be obtained.

Step 7: Termination conditions. In ESMR problem, the optimal solution cannot be obtained in advance, therefore maximum evolution algebra should be given as a termination conditions. Based on [20], define the termination function $u_{F}=\left|\frac{F_{n+1}-F_{n}}{F_{n}}\right|$, and $u_{f}=\left|\frac{f_{n+1}-f_{n}}{f_{n}}\right|$. If $\max \left(u_{F}, u_{f}\right) \leq \tau$, then the algorithm is convergent. Here, $\tau$ means the iteration accuracy.

\section{CASE STUDY}

Wind turbine generator system is a complex product, which usually contains 41 production cells. Each cell can be regarded as a production task. Taking the scheduling of manufacturing equipment resource as example, equipment information is shown in Table I. For a $5 \mathrm{MW}$ product, the relationship of task-equipment is shown in Table II. To cope with the possible emergencies, task's importance should be evaluated firstly. Relationship of production tasks includes process products supply $\left(r_{1}\right)$, manufacturing information transfer $\left(r_{2}\right)$ and manufacturing resource share $\left(r_{3}\right)$. According to Eqs. (16) to (19), the linguistic variable and triangular fuzzy numbers can be calculated, as shown in Table III. Then, the relationship between tasks can be determined by Eq. (21). Thus, the CNPS of product A can be illustrated as shown in Fig. 6.

Table I: Equipment information.

\begin{tabular}{|l|c|c|c|}
\hline \multicolumn{1}{|c|}{ Name } & Number & Cost-in-use & Code \\
\hline Crane $(100 \mathrm{t})$ & 1 & 3600 & 1 \\
Crane $(60 \mathrm{t})$ & 2 & 2100 & $2-3$ \\
Crane $(32 \mathrm{t})$ & 1 & 1200 & 4 \\
Crane $(10 \mathrm{t})$ & 8 & 1000 & $5-12$ \\
Flatbed & 2 & 160 & $13-14$ \\
Electric forklift (2t) & 2 & 6 & $15-20$ \\
Hand fork lifter & 10 & 1 & $21-30$ \\
Electromagnetic heater & 2 & 120 & $31-32$ \\
Auxiliaries & 50 & 0.5 & $33-82$ \\
\hline
\end{tabular}


Table II: Relationship of task-equipment.

\begin{tabular}{|c|c|c|c|c|c|c|c|c|c|c|c|c|c|}
\hline 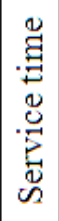 & 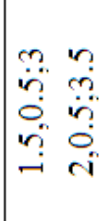 & $\begin{array}{l}0 \\
0 \\
\ddot{n} \\
\stackrel{-}{-} \\
\stackrel{-}{i} \\
\ddot{i}\end{array}$ & 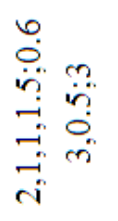 & $\begin{array}{l}m \\
\ddot{n} \\
0 \\
n \\
m\end{array}$ & 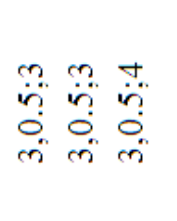 & $\begin{array}{l}n \\
\ddot{n} \\
0 \\
m\end{array}$ & $\begin{array}{l}m \\
m \\
m \\
m\end{array}$ & $\begin{array}{l}m \\
m \\
m \\
m \\
m\end{array}$ & $\begin{array}{l}\text { ra } \\
\text { in } \\
\text { iv }\end{array}$ & $\begin{array}{l}\text { ci } \\
\text { ci } \\
\text { ci }\end{array}$ & $\begin{array}{l}\stackrel{9}{-} \\
6 \\
\stackrel{0}{0} \\
\dot{i}\end{array}$ & $\begin{array}{l}\rightrightarrows \\
\Rightarrow \\
-\end{array}$ & $\begin{array}{l}\Rightarrow \\
\Rightarrow \\
\Rightarrow\end{array}$ \\
\hline 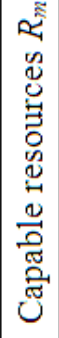 & 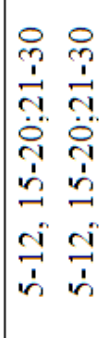 & 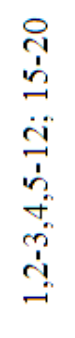 & 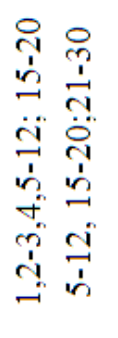 & 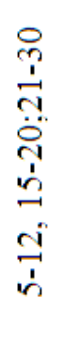 & 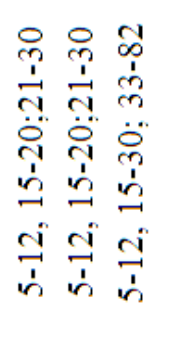 & 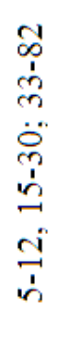 & ri & 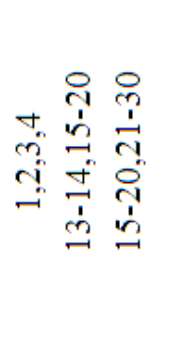 & $\begin{array}{l}+ \\
\stackrel{n}{\sim} \\
\stackrel{-}{-}\end{array}$ & 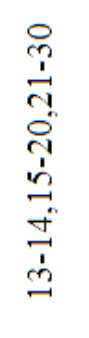 & 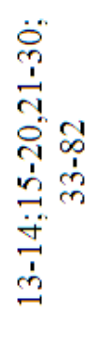 & 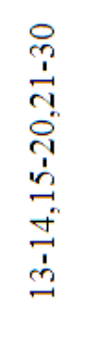 & 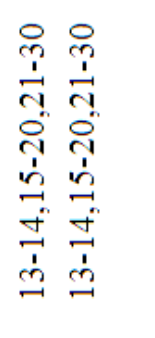 \\
\hline 5 & n & 2 & 68 & "n & $\stackrel{9}{8} \%$ & ñ & 요 & $m \circ 6$ & $\infty$ & $\stackrel{6}{2}$ & 2 & $n$ & $n \infty$ \\
\hline 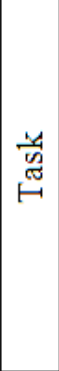 & 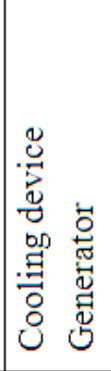 & 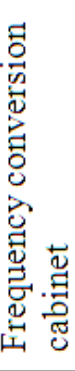 & 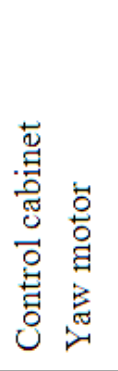 & 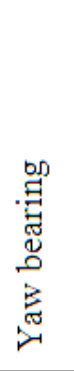 & 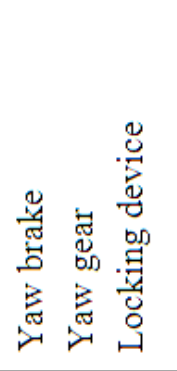 & 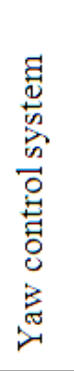 & 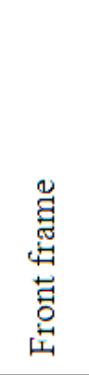 & 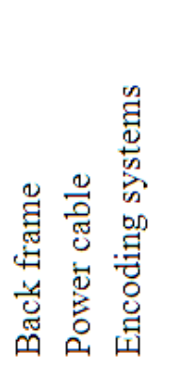 & 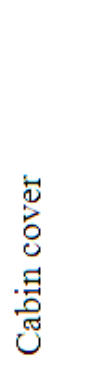 & 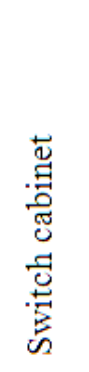 & 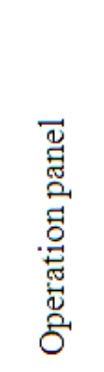 & 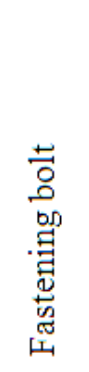 & 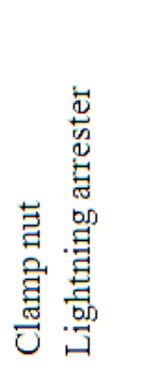 \\
\hline$\dot{0}$ & $\sqrt{2} d$ & $\stackrel{4}{4}$ & $a \ddot{c}$ & $\vec{c}$ & ભ શે & $\vec{m}$ & लै & $m+n$ & $\begin{array}{l}b \\
n\end{array}$ & $n$ & $\stackrel{\infty}{m}$ & ले & 웤 \\
\hline 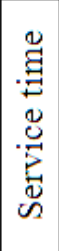 & & 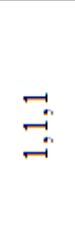 & 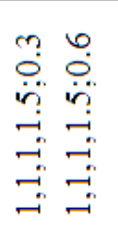 & 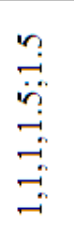 & 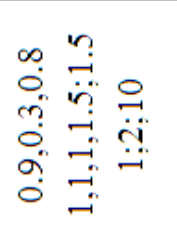 & 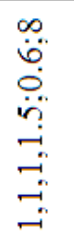 & $\begin{array}{l}\infty \\
\ddot{6} \\
0 \\
\ddot{n} \\
- \\
ت \\
- \\
-\end{array}$ & 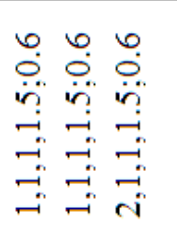 & 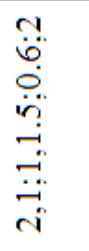 & 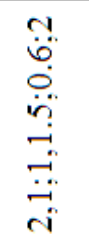 & 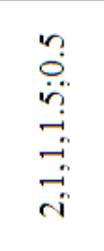 & 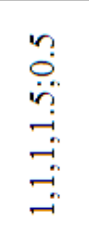 & 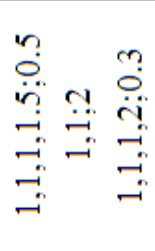 \\
\hline 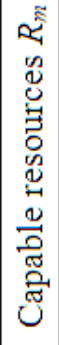 & & $\begin{array}{c}m \\
i \\
i\end{array}$ & 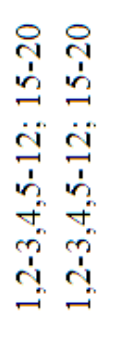 & 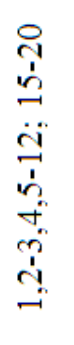 & 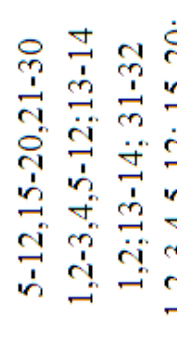 & 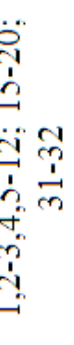 & 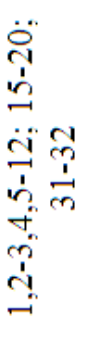 & 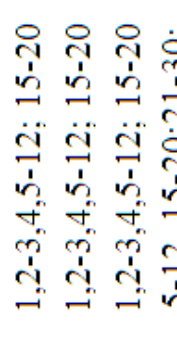 & 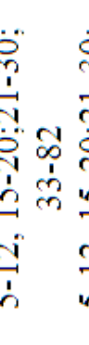 & 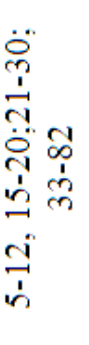 & 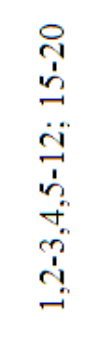 & 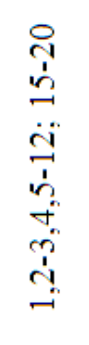 & 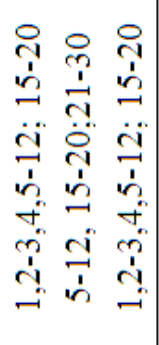 \\
\hline 点 & 28 & in & $n$ m & $m$ & "n n 우 & $n$ & in & 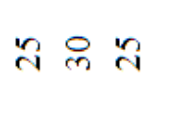 & 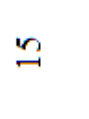 & $n$ & $ㅇ$ & $\stackrel{9}{+}$ & $\begin{array}{c}0 \\
\cdots\end{array}$ \\
\hline 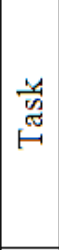 & 藏 & 㞼 & 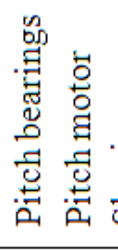 & $\frac{1}{2}$ & 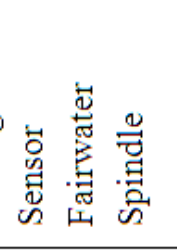 & 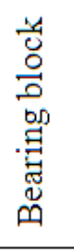 & م. & 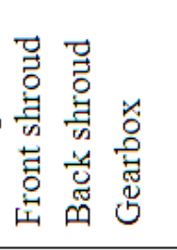 & 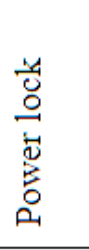 & 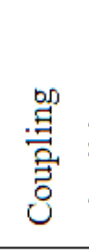 & 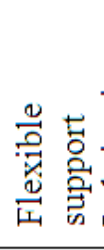 & 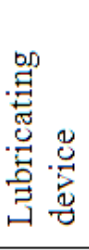 & 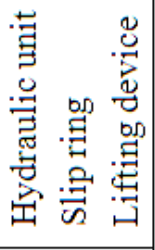 \\
\hline 只 & $-a$ & $m$ & $\forall n$ & 6 & $n \infty a$ & $ㅇ$ & $\exists$ & \pm & $\stackrel{n}{n}$ & $\stackrel{0}{-}$ & $\Xi$ & $\stackrel{\infty}{-}$ & $\stackrel{ }{\rightarrow} \vec{\imath}$ \\
\hline
\end{tabular}


Table III: Linguistic variable and triangular fuzzy numbers.

\begin{tabular}{|l|c|c|c|}
\hline Linguistic variable & $\varphi_{i j, \kappa}^{L}$ & $\varphi_{i j, \kappa}^{M}$ & $\varphi_{i j, \kappa}^{R}$ \\
\hline Infinitesimally small & 0 & 0 & 0.1667 \\
Smaller & 0 & 0.1667 & 0.3333 \\
Small & 0.1667 & 0.3333 & 0.5 \\
Ordinary & 0.3333 & 0.5 & 0.6667 \\
Large & 0.5 & 0.6667 & 0.8333 \\
Larger & 0.6667 & 0.8333 & 1 \\
Extremely large & 0.8333 & 1 & 1 \\
\hline
\end{tabular}

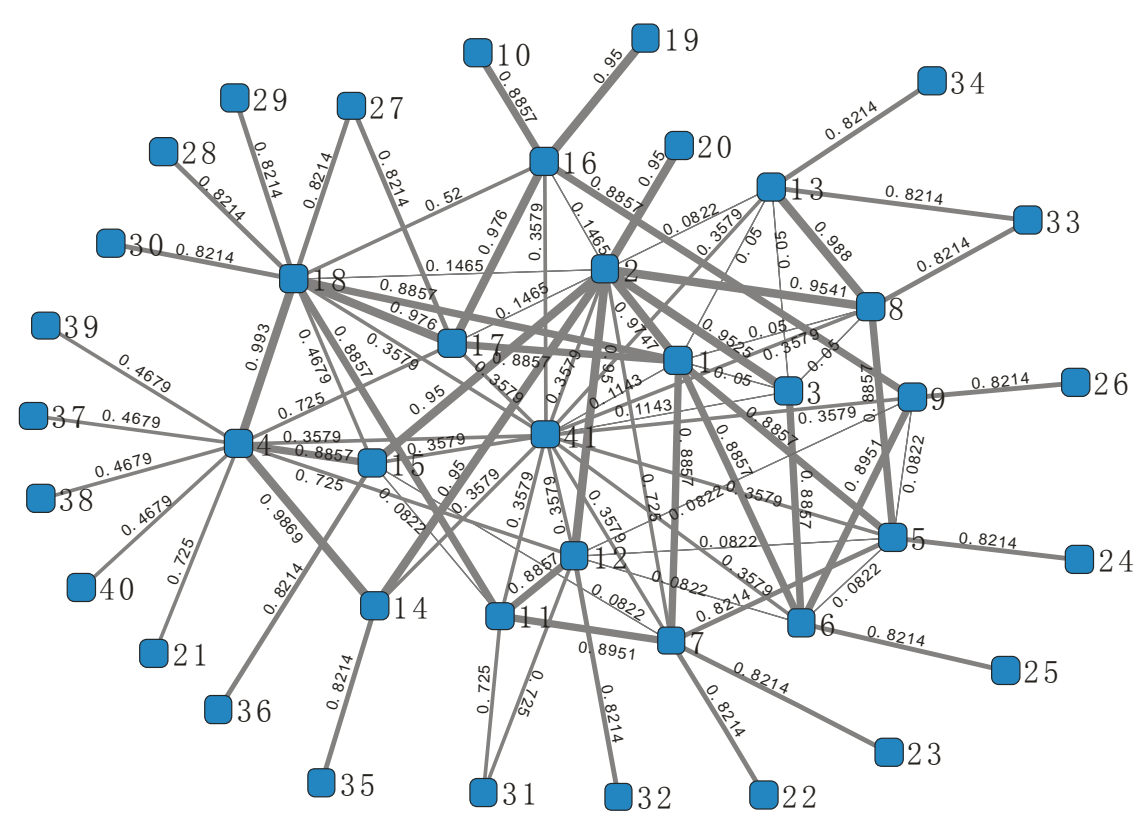

Figure 6: Topology of CNPS.

Based on the task importance evaluation method in Section 3.1, the vulnerability should be analysed. According to Eqs. (14) to (21), the vulnerability, can be calculated. Then we sort the results in descending order, as shown in Table IV, which is just the task importance order.

At $t=65$, there is a fault in device 4 , and it will recover to normal at $t=85$. Besides, due to market environment changes, customer requests to advance the delivery to 200 from 235 . According to contract terms and actual production progress, $\beta=0.8$, popsize $=200$, the maximum generation is $500, \lambda_{c 1}=0.8, \lambda_{c 2}=0.7, \lambda_{m 1}=0.01, \lambda_{m 2}=0.001, \tau=0.00001$. On this occasion, results of ESMR calculated by Matlab R2010a are shown in Fig. 7 and Table V. In Fig. 7, the fitness values change curves of AACA [21], SAGA [22], MOGA[12] and AMODRAA are illustrated, and obviously the method proposed in this paper has a faster convergence and better precision. Under this ESMR scheme, resources tardiness is as shown in Fig. 8, most resources are arrival before the latest time, which greatly reduces the influence.

To further analyse the influence of task invalidation to production system, suppose each task break down once time, and run AMODRAA 50 times based on the above steps for each failure, then 41 ESMR schemes can be reached. By taking task importance, average fitness value and task number as left ordinate, right ordinate and abscissas respectively, the relationship of task importance and the objective function value is illustrated in Fig. 9, in which these two are positively related. The results declare that the more important task, the greater impact on production system when the task in failure. Thus, under the emergency, the tasks with a higher importance should be guaranteed in normal. 
Table IV: Vulnerability-based task importance.

\begin{tabular}{|c|c|c||c|c|c||c|c|c|}
\hline Number & $v_{i}$ & $V\left(G, v_{i}\right)$ & Number & $v_{i}$ & $V\left(G, v_{i}\right)$ & Number & $v_{i}$ & $V\left(G, v_{i}\right)$ \\
\hline 1 & $v_{9}$ & $21.01 \%$ & 15 & $v_{18}$ & $7.85 \%$ & 29 & $v_{24}$ & $4.68 \%$ \\
2 & $v_{3}$ & $20.31 \%$ & 16 & $v_{21}$ & $6.78 \%$ & 30 & $v_{25}$ & $4.65 \%$ \\
3 & $v_{5}$ & $17.89 \%$ & 17 & $v_{16}$ & $6.69 \%$ & 31 & $v_{35}$ & $4.61 \%$ \\
4 & $v_{4}$ & $16.35 \%$ & 18 & $v_{20}$ & $6.54 \%$ & 32 & $v_{8}$ & $4.58 \%$ \\
5 & $v_{14}$ & $15.38 \%$ & 19 & $v_{7}$ & $6.43 \%$ & 33 & $v_{6}$ & $4.55 \%$ \\
6 & $v_{32}$ & $14.64 \%$ & 20 & $v_{19}$ & $5.49 \%$ & 34 & $v_{40}$ & $4.51 \%$ \\
7 & $v_{33}$ & $11.17 \%$ & 21 & $v_{29}$ & $5.30 \%$ & 35 & $v_{41}$ & $4.42 \%$ \\
8 & $v_{11}$ & $10.43 \%$ & 22 & $v_{30}$ & $5.24 \%$ & 36 & $v_{36}$ & $4.39 \%$ \\
9 & $v_{12}$ & $10.33 \%$ & 23 & $v_{15}$ & $4.98 \%$ & 37 & $v_{38}$ & $3.37 \%$ \\
10 & $v_{13}$ & $10.01 \%$ & 24 & $v_{17}$ & $4.97 \%$ & 38 & $v_{37}$ & $3.29 \%$ \\
11 & $v_{10}$ & $9.96 \%$ & 25 & $v_{22}$ & $4.91 \%$ & 39 & $v_{39}$ & $3.27 \%$ \\
12 & $v_{23}$ & $9.87 \%$ & 26 & $v_{28}$ & $4.88 \%$ & 40 & $v_{2}$ & $2.82 \%$ \\
13 & $v_{27}$ & $9.65 \%$ & 27 & $v_{31}$ & $4.82 \%$ & 41 & $v_{1}$ & $2.66 \%$ \\
14 & $v_{26}$ & $9.57 \%$ & 28 & $v_{34}$ & $4.78 \%$ & & & \\
\hline
\end{tabular}

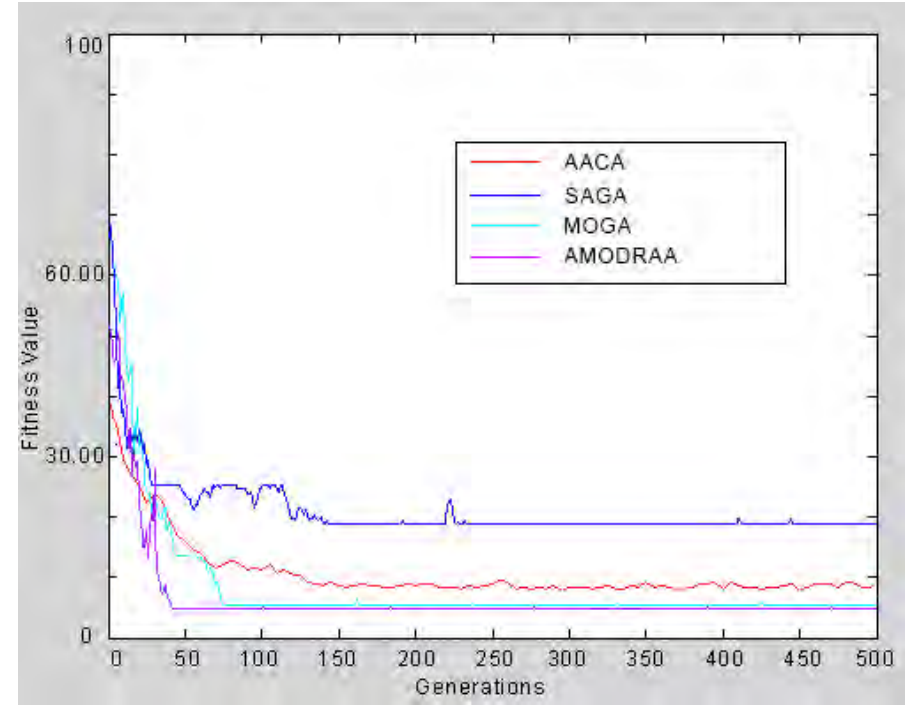

Figure 7: Fitness values change curves.

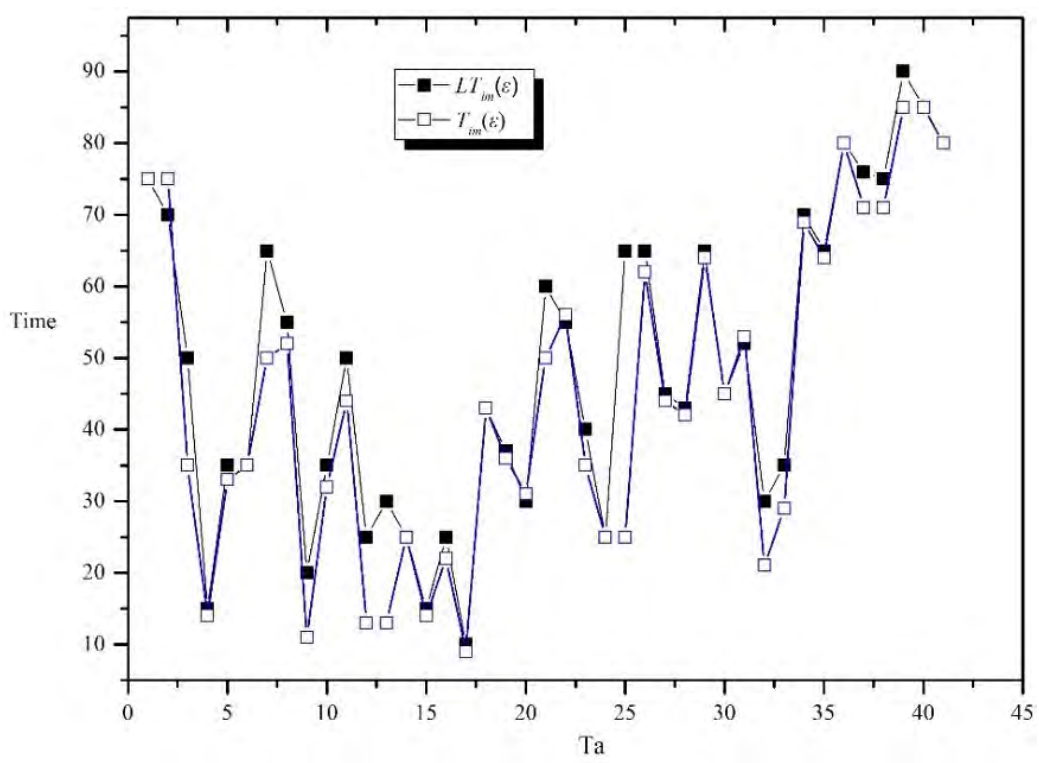

Figure 8: Resources tardiness under ESMR. 
Table V: ESMR scheme.

\begin{tabular}{|c|c|c|c|c|c|}
\hline No. & Task & Resource $R_{m}$ & No. & Task & Resource $R_{m}$ \\
\hline 1 & Vane & - & 22 & Cooling device & $15 ; 24$ \\
\hline 2 & Tower & - & 23 & Generator & $11 ; 25$ \\
\hline 3 & Hub & 3 & 24 & Frequency conversion cabinet & $12 ; 15$ \\
\hline 4 & Pitch bearings & $12 ; 15$ & 25 & Control cabinet & $13 ; 16$ \\
\hline 5 & Pitch motor & $11 ; 16$ & 26 & Yaw motor & $5 ; 21$ \\
\hline 6 & Slewing bearing & $10 ; 15$ & 27 & Yaw bearing & $6 ; 22$ \\
\hline 7 & Sensor & 16,21 & 28 & Yaw brake & $7 ; 23$ \\
\hline 8 & Fairwater & $4 ; 13$ & 29 & Yaw gear & $8 ; 24$ \\
\hline 9 & Spindle & $2 ; 14 ; 31$ & 30 & Locking device & $15 ; 35$ \\
\hline 10 & Bearing block & $12 ; 17 ; 32$ & 31 & Yaw control system & $16 ; 36$ \\
\hline 11 & Spindle bearing & $11 ; 1 ; 31$ & 32 & Front frame & 1 \\
\hline 12 & Front shroud & $5 ; 15$ & 33 & Back frame & 1 \\
\hline 13 & Back shroud & $6 ; 18$ & 34 & Power cable & 14 \\
\hline 14 & Gearbox & $4 ; 15$ & 35 & Encoding systems & 21 \\
\hline 15 & Power lock & $20 ; 22 ; 33$ & 36 & Cabin cover & 3 \\
\hline 16 & Coupling & $16 ; 23 ; 34$ & 37 & Switch cabinet & 22 \\
\hline 17 & Flexible support & $7 ; 17$ & 38 & Operation panel & 23 \\
\hline 18 & $\begin{array}{l}\text { Lubricating } \\
\text { device }\end{array}$ & $12 ; 15$ & 39 & Fastening bolt & 24 \\
\hline 19 & Hydraulic unit & $7 ; 18$ & 40 & Clamp nut & 24 \\
\hline 20 & Slip ring & $8 ; 19$ & 41 & Lightning arrester & 25 \\
\hline 21 & Lifting device & $4 ; 20$ & & & \\
\hline
\end{tabular}

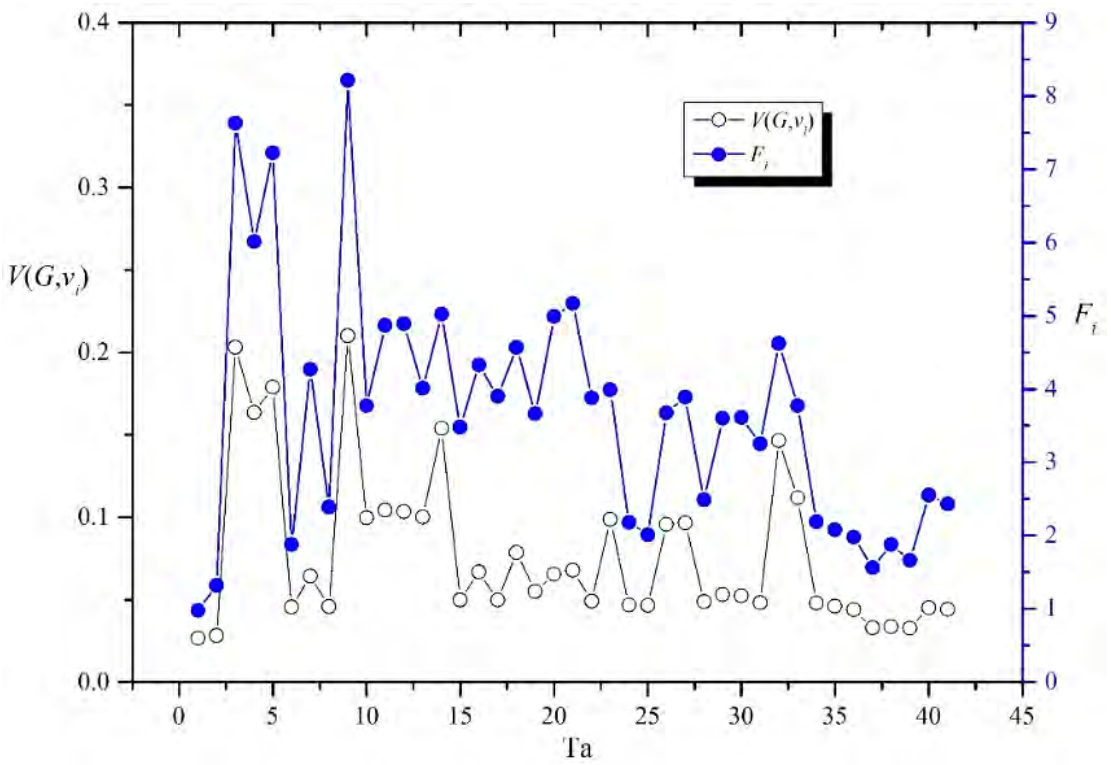

Figure 9: Relationship of task importance and the objective function value.

\section{CONCLUSIONS}

To reduce the influence of incident to the production system, this paper studies the emergency decision-making method for manufacturing resources scheduling, which includes a mathematical model, the rolling optimization of resources scheduling method, vulnerabilitybased task importance evaluation, an adaptive multi-objective dynamic resource scheduling algorithm and a case of Wind turbine generator system. The research results show that this 
method is not only able to effectively reduce the complexity of ESMR, but also to reveal influence of task importance on ESMR decision-making. Note that the vulnerability-based method for evaluating the task importance shows better results than traditional methods. The scheme we attained has a greater global optimality. With the analysis, it can provide way of measuring the overall emergency impacts on the systems, and allow the decision-makers to respond to unexpected events that what the best way of resources scheduling is.

ESMR is an extremely complex and dynamic optimization problem. The research of this paper is based on that all manufacturing resources during the whole process is deterministic, and not consider the fuzziness and uncertainties of resource and production system. Thus, the research in future will focus on the ESMR under uncertainties and fuzziness.

\section{ACKNOWLEDGMENTS}

This research is sponsored by National Natural Science Foundation of China under Project number 71571023, 71401019. We are grateful for the constructive suggestions provided by the reviewers, which would improve the paper.

\section{REFERENCES}

[1] Eckert, C.; Clarkson, P. J; Zanker, W. (2004). Change and customisation in complex engineering domains, Research in Engineering Design, Vol. 15, No. 1, 1-21, doi:10.1007/s00163-003-0031-7

[2] Johansen, S. G.; Thorstenson, A. (1998). An inventory model with Poisson demands and emergency orders, International Journal of Production Economics, Vol. 56-57, 275-289, doi:10.1016/S0925-5273(97)00126-6

[3] Gokpinar, B.; Hopp, W. J.; Iravani, S. M. R. (2010). The impact of misalignment of organizational structure and product architecture on quality in complex product development, Management Science, Vol. 56, No. 3, 468-484, doi:10.1287/mnsc.1090.1117

[4] Braha, D.; Bar-Yam, Y. (2007). The statistical mechanics of complex product development: Empirical and analytical results, Management Science, Vol. 53, No. 7, 1127-1145, doi: $10.1287 / \mathrm{mnsc} .1060 .0617$

[5] Jin, F.; Wu, C. (2006). Research status and prospects for massive production scheduling, Computer Integrated Manufacturing Systems, Vol. 12, No. 2, 161-168

[6] Xia, Y. S.; Yang, M.-H.; Golany, B.; Gilbert, S. M.; Yu, G. (2004). Real-time disruption management in a two-stage production and inventory system, IIE Transactions, Vol. 36, No. 2 , 111-125, doi:10.1080/07408170490245379

[7] Xiao, T.; Qi, X. (2008). Price competition, cost and demand disruptions and coordination of a supply chain with one manufacturer and two competing retailers, Omega, Vol. 36, No. 5, 741753, doi:10.1016/j.omega.2006.02.008

[8] Qi, X.; Bard, J. F.; Yu, G. (2004). Supply chain coordination with demand disruptions, Omega, Vol. 32, No. 4, 301-312, doi:10.1016/j.omega.2003.12.002

[9] Jia, H.-Z.; Ordonez, F.; Dessouky, M. M. (2007). Solution approaches for facility location of medical supplies for large-scale emergencies, Computers \& Industrial Engineering, Vol. 52, No. 2, 257-276, doi:10.1016/j.cie.2006.12.007

[10] Abreu, C. F.; May, J. H.; Spangler, W. E.; Vargas, L. G. (2008). Conflict identification and reconciliation in a collaborative manufacturing scheduling task, International Journal of Information Technology \& Decision Making, Vol. 7, No. 1, 147-174, doi:10.1142/ S0219622008002855

[11] Zhang, Z.-L.; Li, X.-Y.; Zhang, Z.-Q. (2009). Emergency product production based on production capacity reserve, Computer Integrated Manufacturing Systems, Vol. 15, No. 7, 1349$1353(1373)$

[12] Liang, C.; Guo, J.; Yang, Y. (2011). Multi-objective hybrid genetic algorithm for quay crane dynamic assignment in berth allocation planning, Journal of Intelligent Manufacturing, Vol. 22, No. 3, 471-479, doi:10.1007/s10845-009-0304-8 
[13] Amankwah, H.; Larsson, T.; Textorius, B. (2014). A maximum flow formulation of a multiperiod open-pit mining problem, Operational Research, Vol. 14, No. 1, 1-10, doi:10.1007/ s12351-013-0140-7

[14] Chen, J.-Y.; Lin, Q.-Z.; Hu, Q.-B. (2010). Application of novel clonal algorithm in multiobjective optimization, International Journal of Information Technology \& Decision Making, Vol. 9, No. 2, 239-266, doi:10.1142/S0219622010003804

[15] Ramirez-Marquez, J. E.; Rocco, C. M. (2012). Vulnerability based robust protection strategy selection in service networks, Computers \& Industrial Engineering, Vol. 63, No. 1, 235-242, doi: $10.1016 /$ j.cie.2012.02.016

[16] Doumeingts, G.; Chen, D.; Marcotte, F. (1992). Concepts, models and methods for the design of production management systems, Computers in Industry, Vol. 19, No. 1, 89-111, doi:10.1016/ 0166-3615(92)90009-C

[17] Yen, K. K.; Ghoshray, S.; Roig, G. (1999). A linear regression model using triangular fuzzy number coefficients, Fuzzy Sets and Systems, Vol. 106, No. 2, 167-177, doi:10.1016/S01650114(97)00269-8

[18] Opricovic, S.; Tzeng, G.-H. (2003). Defuzzification within a multicriteria decision model, International Journal of Uncertainty, Fuzziness and Knowledge-Based Systems, Vol. 11, No. 5, 635-652, doi:10.1142/S0218488503002387

[19] Felea, I.; Dzitac, S.; Vesselenyi, T.; Dzitac, I. (2014). Decision support model for production disturbance estimation, International Journal of Information Technology \& Decision Making, Vol. 13, No. 3, 623-647, doi:10.1142/S0219622014500576

[20] Yu, G. D.; Yang, Y.; Zhao, X.; Li, G. (2014). Multi-objective rescheduling model for product collaborative design considering disturbance, International Journal of Simulation Modelling, Vol. 13, No. 4, 472-484, doi:10.2507/IJSIMM13(4)CO17

[21] Prakash, A.; Tiwari, M. K.; Shankar, R. (2008). Optimal job sequence determination and operation machine allocation in flexible manufacturing systems: an approach using adaptive hierarchical ant colony algorithm, Journal of Intelligent Manufacturing, Vol. 19, No. 2, 161-173, doi:10.1007/s10845-008-0071-y

[22] Yeh, W.-C.; Lai, P.-J.; Lee, W.-C.; Chuang, M.-C. (2014). Parallel-machine scheduling to minimize makespan with fuzzy processing times and learning effects, Information Sciences, Vol. 269, 142-158, doi:10.1016/j.ins.2013.10.023 